\title{
Compras Públicas no Brasil: Tendências de inovação, avanços e dificuldades no período recente
}

\author{
Campos Christo Fernandes, Ciro \\ Compras Públicas no Brasil: Tendências de inovação, avanços e dificuldades no período recente \\ Administração Pública e Gestão Social, vol. 11, núm. 4, 2019 \\ Universidade Federal de Viçosa, Brasil \\ Disponivel em: http://www.redalyc.org/articulo.oa?id=351560525006
}

Esta obra está bajo una Licencia Creative Commons Atribución-NoComercial-SinDerivar 3.0 Internacional. 


\title{
Compras Públicas no Brasil: Tendências de inovação, avanços e dificuldades no período recente
}

\author{
Public Procurement in Brazil: Innovation trends, advances and difficulties in the recent period \\ Contratación pública en Brasil: Tendencias de innovación, avances y dificultades en el período reciente \\ Ciro Campos Christo Fernandes \\ Escola Nacional de Administração Pública, Brasil \\ Redalyc: http://www.redalyc.org/articulo.oa? \\ id $=351560525006$
}

ciro.fernandes@uol.com.br

Recepção: 31 Janeiro 2019

Aprovação: 14 Agosto 2019

Publicado: 01 Outubro 2019

\section{Resumo:}

Este artigo é um balanço dos avanços, limitações e perspectivas para o fortalecimento das compras públicas na Administração Pública brasileira. A pesquisa se baseou no levantamento, categorização e análise de trabalhos sobre temas de compras públicas, focalizando a produção científica apresentada nos congressos anuais de Administração Pública entre 2008 e 2016, promovidos pela associação que congrega os líderes responsáveis pela gestão pública dos estados brasileiros. Uma amostra de trabalhos que tratam especificamente de compras públicas foi selecionada. Apresenta-se uma visão sistemática das principais mudanças e inovações, destacando as seguintes tendências: Marco legal e regulatório, sistemas informatizados e portais na internet, reestruturação dos formatos de compra e contratação, terceirização, metodologias e ferramentas de gestão das compras, uso do poder de compra, ciclo do suprimento, centralização das compras e institucionalização da área.

PalaVRas-CHAVE: licitações, contratações públicas, gestão pública.

\section{Abstract:}

This paper is an assessment of the advances, limitations and prospects for strengthening public procurement in central government and in the Brazilian states (subnational governments). The research was based on a document survey, categorization and analysis of the scientific production of works on public procurement issues. The research focused the scientific production presented at the annual congresses of Public Administration between 2008 and 2016, promoted by the association that congregates the leaders responsible for the public management of Brazilian states. A sample of works that deal specifically with public procurement was selected. A systematic view of the main changes and innovations is presented, stressing the following trends: Legal and regulatory framework, computerized systems and portals on the internet, restructuring of purchase and contracting conformation, outsourcing, methodologies and tools for purchasing management, use of purchasing power, supplies cycle, centralization of purchases and institutionalization of the area.

KEYWORDS: bidding, public contracts, public management.

\section{ReSUMEN:}

Este artículo es un balance de los avances, limitaciones y perspectivas para el fortalecimiento de la contratación pública en la Administración Pública de Brasil. La investigación se basó en la categorización y análisis de obras sobre temas de contratación pública, centrándose en la producción científica presentada en los congresos anuales de Administración Pública entre los años de 2008 y 2016, promovida por la asociación que reúne a los líderes responsables de la gestión pública de los estados brasileños. Se seleccionó una muestra de trabajos que se ocupan específicamente de la contratación pública. Se presenta una visión general sistemática de los cambios e innovaciones clave, destacando las siguientes tendencias: Marco legal y regulatorio, sistemas computarizados y portales de internet, reestructuración de formatos de compra y contratación, tercerización, metodologías y herramientas de gestión de compras, uso del poder adquisitivo, ciclo de suministro, centralización de compras e institucionalización del área.

Palabras ClaVe: licitaciones públicas, contratación pública, gestión pública. 


\section{INTRODUÇÃo}

Este artigo é um balanço dos avanços, limitações e perspectivas para o fortalecimento das compras públicas no governo federal e nos Estados brasileiros, no período recente. A pesquisa foi baseada no levantamento de documentos, categorização e análise da produção de trabalhos sobre temas de compras públicas. Esta é uma área de pesquisa emergente que, nos últimos anos, tem atraído um número crescente de profissionais envolvidos com projetos de inovação nas regras, sistemas, processos e formatos de organização das compras e da logística pública.

A Administração Pública brasileira passou por importantes mudanças na gestão de compras públicas desde a aprovação de uma lei geral de licitações (Lei 8.666), em 1993, e a introdução das tecnologias de informação e do comércio eletrônico nos processos de compras e transações entre o Estado e seus fornecedores. No entanto, essas mudanças ainda não foram objeto de uma avaliação mais abrangente que identifique quais têm sido as tendências de inovação e a extensão da disseminação dessas inovações, particularmente entre os governos estaduais. Além disso, há a necessidade de identificar obstáculos, restrições e questões que dificultam o progresso na área, particularmente, seu desenvolvimento técnico e consolidação institucional.

A perspectiva teórica que fundamenta esta pesquisa considera as abordagens sobre a institucionalização das compras e contratações públicas como uma função administrativa, inserida na construção do estado e da administração pública (Thai, 2001; 2009; Knight et al., 2007; Nagle, 1999). No caso específico do Brasil, essa construção é um processo ainda incompleto que resulta em heterogeneidades, assimetrias e desequilíbrios entre funções, organizações, processos e sistemas que também afetam o relacionamento com os fornecedores (Fernandes, 2014; Fiuza \& Medeiros, 2014). Diante destas circunstâncias, a construção do arcabouço legal e de uma burocracia profissional, assim como a assimilação de capacidades técnicas, o desenvolvimento de sistemas informatizados e o fortalecimento institucional da área são requisitos fundamentais.

A pesquisa analisou os trabalhos apresentados nos congressos de Administração Pública entre 2008 e 2016, promovidos pelo Conselho Nacional de Secretários de Estado de Administração (CONSAD), associação que reúne os líderes responsáveis pela gestão pública dos estados brasileiros. Os Congressos CONSAD de Gestão Pública são realizados anualmente, reunindo cerca de 2.000 participantes a cada evento, em sua maioria servidores públicos dos três níveis de governo. A maior parte dos trabalhos foi produzida por practitioners das administrações públicas estaduais e federal, o que se reflete nas experiências relatadas ao longo deste artigo. Tratam-se de servidores públicos diretamente envolvidos com atividades técnicas ou gerenciais, mas empenhados na sistematização de informações e na reflexão a partir da sua experiência prática.

Levantamentos similares que complementam os resultados obtidos com essa pesquisa foram realizados por Marini (2008) e Dezolt e Barbosa (2016) a partir de dados produzidos diretamente pelos governos estaduais e por Campos (2008), Prado (2014) e Oshiro, Renda dos Santos e Jesus-Lopes (2016), que analisaram a produção sobre compras públicas publicada em revistas científicas ou apresentada em congressos de cunho acadêmico.

\section{TENDÊNCIAS DE MUdANÇA E INOVAÇÃo}

Este artigo pretende contribuir com a construção de uma visão abrangente e sistemática das compras públicas no Brasil, baseada na categorização das principais mudanças e inovações que se disseminaram no período recente. A amostra selecionada contemplou 70 trabalhos que tratam especificamente de compras públicas, contendo pesquisas, diagnósticos, estudos de caso e avaliações de projetos e iniciativas inovadoras na área. Os trabalhos foram coletados entre janeiro e junho de 2017, no repositório do CONSAD (http:// banco.consad.org.br), que publica a produção dos Congressos. Uma versão preliminar deste artigo foi apresentada em sessão realizada no X Congresso, em 6 de julho do mesmo ano. Eventuais lacunas de 
publicação da versão escrita de algum trabalho apresentado nos Congressos são uma possível limitação da coleta realizada. O método de seleção foi a identificação do assunto nos títulos, palavras-chave e resumos.

Uma categorização preliminar foi utilizada para a análise dos trabalhos, baseada em levantamentos internacionais recentes da produção científica sobre compras públicas (Flynn \& Davis, 2014; Patrucco, Luzzini \& Ronchi, 2017) e na sistematização de tendências, experiências e práticas internacionais (Thai \& Grimm, 2000; Thai, 2009; Dimitri, Piga \& Spagnolo, 2006) e dos governos estaduais brasileiros (Dezolt et al., 2016), além do trabalho de MacManus (1996), que descreve os componentes dos sistemas de compras públicas no contexto das transformações que têm atingido essa área.

A categorização foi ajustada de forma interativa, no decorrer do exame dos trabalhos selecionados, para chegar à sistematização final de um conjunto de temas que situasse as mudanças e inovações dentro da perspectiva estratégica de institucionalização da função compras públicas no Brasil. Dessa forma, o balanço apresentado destaca não somente a frequência dos temas que foram objeto de experiências inovadoras, muitas delas alcançando disseminação nacional, como também as deficiências e limitações que indicam possíveis itens de uma agenda para o fortalecimento da área.

Assim, o levantamento levou à identificação das seguintes tendências de transformação das compras públicas: Arcabouço legal e normativo, sistemas informatizados e portais na internet, reestruturação dos formatos de compra e contratação, terceirização, metodologias e ferramentas de gestão das compras, uso do poder de compra, ciclo do suprimento, centralização das compras e institucionalização da função compras e contratações públicas.

A Tabela 1 apresenta a distribuição dos trabalhos examinados, por tema e escopo geográfico, permitindo uma visão do conjunto da produção sobre compras públicas. O escopo geográfico se refere à Administração Federal (União) ou ao estado da federação objeto de algum trabalho. Quando se refere a mais de um Estado, por exemplo, na forma de análise comparativa, o escopo geográfico é definido como "estados".

O arcabouço legal e normativo das compras e contratações foi explorado em 9 trabalhos que trataram da estrutura normativa no âmbito federal e dos estados e das experiências de criação e implantação do pregão, do registro de preços e do Regime Diferenciado de Contratações (RDC). Os sistemas informatizados de apoio aos processos de compra e contratação e portais de compras públicas na internet conformaram um segundo grupo temático, com 7 trabalhos voltados para as experiências estaduais e da Administração Federal.

A reestruturação dos formatos de compra e contratação foi o tema de 15 trabalhos sobre experiências de implantação dos cartões de crédito corporativos para suprimento de fundos, cotação eletrônica para compras de pequeno valor, acordos de nível de serviços e novos formatos de contratação em telecomunicações, compra de passagens e abastecimento de frotas. A terceirização foi o objeto específico de 8 trabalhos que discutiram sua aplicação e os mecanismos e metodologias para contratação e gestão de serviços. O tema das metodologias e ferramentas de gestão das compras foi abordado por 14 trabalhos que trataram do planejamento das compras, padronização de materiais e serviços, estimação de preços de referência, metodologias e sistemas de controle de custos e avaliação de fornecedores.

O uso do poder de compra do estado recebeu 9 trabalhos que analisaram aspectos legais e normativos das regras de preferência e experiências estaduais de políticas de fomento às micro e pequenas empresas e de sustentabilidade ambiental. O ciclo do suprimento foi abordado em 3 trabalhos com análises gerais e sobre a Administração Federal e os estados. A centralização das compras como modelo de organização foi analisada em 3 trabalhos. Finalmente, a discussão sobre a institucionalização da função compras e contratações na administração pública foi tema de 2 trabalhos que abordaram a Administração Federal e os estados.

A distribuição por escopo geográfico dos trabalhos evidencia a representatividade da produção nacional sobre compras públicas, que focalizou 15 estados de todas as regiões do país, além da Administração Federal e municípios. A distribuição, considerando somente os trabalhos direcionados especificamente a alguma experiência estadual, perfaz um total de 35 trabalhos que exploram 7 do total de 9 temas. As experiências de Minas Gerais e São Paulo foram responsáveis pela maior participação na produção, com 11 trabalhos. As 
abordagens que apresentam levantamentos abrangendo vários estados correspondem a 5 trabalhos, além de 1 trabalho sobre municípios. Abordagens gerais, na forma de análise de questões da área, sem a focalização de alguma experiência específica, foram encontradas em 9 trabalhos. As subseções adiante expandem a análise dos trabalhos, situando-os no âmbito de cada uma das nove tendências identificadas. A análise foi complementada pela contribuição de outros trabalhos publicados em periódicos acadêmicos, quando pertinentes à contextualização ou aprofundamento de algum tema.

Tabela 1 - Distribuição por tema e escopo geográfico

\begin{tabular}{|c|c|c|}
\hline Tema & Quant. & Escopo Geográfico \\
\hline Arcabouço legal e normativo & 9 & $\begin{array}{l}\text { APF, Estados, SP, SE, PE, } \\
\text { CE, PA }\end{array}$ \\
\hline $\begin{array}{l}\text { Sistemas informatizados e portais na } \\
\text { internet }\end{array}$ & 7 & $\begin{array}{l}\text { APF, Estados, RJ, MG, PR, } \\
\text { SP, CE }\end{array}$ \\
\hline $\begin{array}{l}\text { Reestruturação dos formatos de compra e } \\
\text { contratação }\end{array}$ & 15 & $\begin{array}{l}\text { ES, MG, SP, PI, AM, SE, CE, } \\
\text { PA }\end{array}$ \\
\hline Terceirização & 8 & $\mathrm{APF}, \mathrm{SP}, \mathrm{MG}, \mathrm{TO}$ \\
\hline $\begin{array}{l}\text { Metodologias e ferramentas de gestão das } \\
\text { compras }\end{array}$ & 14 & $\begin{array}{l}\text { APF, MG, BA, ES, RS, AM, } \\
\text { SP, PE, RN }\end{array}$ \\
\hline Uso do poder de compra & 9 & APF, PE, SE, RS, SP \\
\hline Ciclo do suprimento & 3 & APF, Estados \\
\hline Centralização das compras & 3 & $\mathrm{APF}, \mathrm{CE}, \mathrm{MG}$ \\
\hline $\begin{array}{l}\text { Institucionalização da função compras e } \\
\text { contrataçốes }\end{array}$ & 2 & APF, Estados \\
\hline Total & 70 & \\
\hline
\end{tabular}

Legenda: APF - Administração Pública Federal

\subsection{Arcabouço legal e normativo}

O arcabouço de leis e normas que regulam as compras e contratações públicas tem sido intensamente afetado pelo movimento internacional de transformação e convergência das regras e procedimentos, impulsionado pelo comércio eletrônico, globalização dos mercados e iniciativas de integração econômica e abertura comercial (Arrowsmith \& Davies, 1998; Arrowsmith, \& Trybus, 2003). No Brasil, a inovação pontual nos procedimentos e a aplicação da tecnologia nas licitações tem sido o caminho de atualização do marco legal e regulatório, ainda pendente de uma revisão abrangente da Lei 8.666/93.

As inovações introduzidas no arcabouço legal e normativo se concentraram em três mudanças-chave cujo alcance e impactos têm sido decisivos para a trajetória da área, com desdobramentos muito amplos, conforme discutido a seguir. São elas o pregão, o registro de preços e, mais recentemente, o novo regime de contratação das obras de engenharia. O pregão foi uma experiência de inovação legal e normativa que, com alguma ousadia, inovou os procedimentos de negociação e seleção de propostas encontrando nos leilões reversos um formato que intensificava a competição e permitia a renegociação dinâmica de propostas, até a seleção da vencedora. A disseminação desse formato alcançou proporções extraordinárias no Brasil. Desde sua criação, em 2000, o pregão se tornou, em poucos anos, a modalidade de licitação mais utilizada, crescendo rapidamente de 3,3\% em 2001 para $72 \%$ do valor total das licitações realizadas em 2014, no âmbito da Administração Federal (Fernandes, 2016).

A criação do pregão resultou do aproveitamento criativo e tempestivo da oportunidade política de decisão de governo, por meio de medida provisória, para atender a uma área até então fechada a experimentações inovadoras que se contrapusessem ao arcabouço legal e normativo construído em torno da Lei 8.666/93 
(Fernandes, op. cit.). A disseminação notável que alcançou se deve, em alguma medida, à simplificação de procedimentos e agilização de prazos, avanços bem aceitos pelos dirigentes e técnicos envolvidos diretamente com as licitações. O pregão atendeu também ao propósito principal da sua criação, que era a redução de custos das compras públicas nas licitações, conforme discutido em trabalhos de pesquisa analisados por Fernandes (op. cit.). Esses trabalhos sugerem ainda possíveis impactos positivos indiretos, atribuíveis ao pregão, como a mudança da cultura da área de compras na Administração Pública, com o empoderamento e consequente valorização do "pregoeiro", a aplicação da tecnologia da informação nas transações com os fornecedores e o impulso a inovações derivadas, como o registro de preços vencedores do pregão. O "pregoeiro" é um servidor público designado para conduzir os pregões, dotado de autonomia e atribuições legais da função que lhe permitem decidir sobre o andamento e o encerramento do rito e a aceitação ou não de recursos interpostos pelos participantes.

Mas há limitações na experiência com o pregão, particularmente o caráter circunscrito e incompleto de que se revestiu a sua criação, como modalidade de uso alternativo que se sobrepunha ao arcabouço legal e normativo das licitações. Teixeira, Prado Filho e Salomão (2016) problematizaram a bem-sucedida disseminação do pregão como inovação que se presta à seleção de propostas pelo menor preço, mas não equaciona aspectos de qualidade e de adequação às necessidades, que depende do planejamento e de estratégias de compra.

Amorim (2016) estudou os resultados da utilização do pregão na contratação de serviços continuados. Identificou um elevado percentual de contratos rescindidos (30\%) devido a falhas atribuíveis a problemas como a saúde financeira das empresas (op. cit.). Como as empresas são levadas à redução de preços das suas propostas, motivadas pelo ambiente competitivo do pregão, os resultados da sua pesquisa indicam a necessidade de discutir a tendência à celebração de contratos com preços inexequíveis.

O registro de preço nas licitações, criado em 2001, tem sido a mais influente inovação sobre a forma de contratação nas licitações e já está atualmente disseminada por todos os níveis de governo. A regulamentação do registro de preços, previsto na Lei 8.666/93, abriu aos governos estaduais a possibilidade de implantar o novo instituto em conexão com as iniciativas de centralização das compras e de introdução de inovações tecnológicas, em especial os portais de compras e o pregão eletrônico.

Em Sergipe (Prado, Zylberman \& Andrade, 2008a) e Pernambuco (Araújo \& Gomes, 2010), o registro de preços apoiou a centralização das contratações, incumbindo ao órgão central de compras o levantamento da demanda e a condução das licitações dos bens e serviços de uso comum entre os órgãos. No Pará, a centralização se apoiou na criação de ferramentas informatizadas que tornaram obrigatória a adesão ao registro de preços no caso de contratações padronizadas e de grande volume (Negrão \& Matos, 2016). No Ceará, o registro de preços também foi implantado ao lado da centralização das compras, como instrumento para sua viabilização, motivado pela necessidade de controle das despesas (Bezerra et al., 2010).

Conforme evidenciado nesses trabalhos, a implantação do registro de preços em alguns estados se deu associada a medidas de controle de custos e despesas e à centralização das compras e contratações. A sua disseminação parece ter sido impulsionada por iniciativas dos dirigentes dos órgãos empenhados em obter as vantagens da concentração das compras para obtenção de preços melhores agilização de prazos. O registro de preços permite contornar a complexidade procedimental da licitação e a debilidade técnica das equipes para atender às necessidades de forma tempestiva, o que o torna atraente para contornar as limitaçóes técnicas e de organização das áreas de compras e contratações. Uma questão a ser considerada é a da utilização do registro de preços como simples artifício para queimar etapas procedimentais poder resultar em perda de eficiência e qualidade das compras e contratações.

Mais recentemente, uma terceira inovação normativa foi a criação, em 2009, de um conjunto de regras específicas para a contratação das obras de engenharia de longa duração, o RDC. O novo regime, criado pela Lei no 12.462 , de 4 de agosto de 2011 , trouxe procedimentos alternativos de negociação e seleção e de contratação, incorporando muitas das inovações procedimentais testadas com o pregão. A sua aprovação se 
deu num contexto da pressão política pelo cumprimento dos prazos dos eventos esportivos internacionais da Copa do Mundo de 2014 e dos Jogos Olímpicos de 2016. Ampliado seu escopo para atender o cronograma das obras prioritárias do governo federal no âmbito do Programa de Aceleração do Crescimento (PAC) e sucessivamente para as obras das áreas de ensino, saúde, portos, aeroportos, silos, unidades prisionais e locais atingidos por desastres naturais, converteu-se em novo marco legal para as obras de engenharia de maior complexidade, criado à margem da Lei 8.666/93.

O RDC reduziu prazos, simplificou regras e introduziu procedimentos de seleção e negociação para incentivar a competição e a assunção dos riscos de execução dos projetos pelos contratados. Conforme mencionado, as suas regras incorporaram os avanços procedimentais do pregão, especialmente a inversão de fases, simplificação procedimental, encurtamento de prazos e utilização dos meios eletrônicos (Oliveira, Pessoa Neto \& Torres, 2013, 2015). Uma importante inovação que acompanhou o desenho aberto com a criação do pregão foi a ruptura com as modalidades de licitação para a escolha entre níveis de flexibilização definidas com base no custo passando a considerar as necessidades de cada contratação e combinando ferramentas e características procedimentais.

\subsection{Sistemas informatizados e portais na internet}

Os sistemas informatizados de apoio aos procedimentos de compra e contratação se desenvolveram e passaram a operar cada vez mais em plataformas abertas à conexão com a internet, para permitir transações com os fornecedores (Neef, 2001). Os sistemas podem potencialmente abranger todo o fluxo das etapas que compõem o ciclo do suprimento (veja seção 2.7), mas as experiências relatadas indicam uma concentração naquelas etapas específicas do processo da licitação stricto sensu, ou seja, oferta e seleção das propostas de compra. A informatização é também uma oportunidade para o redesenho das normas, processos e estruturas organizacionais da área de compras e contratações (Croom \& Brandon-Jones, 2005; Moon, 2005; Mccue \& Roman, 2012). Nesse sentido, o impacto da implantação dos sistemas informatizados pode ser transformador em relação às práticas tradicionais ou se limitar a melhorias incrementais (MacManus, 2002).

Alguns trabalhos analisaram sistemas do Rio de Janeiro (Pacano, Pinto \& Nunes, 2010), Paraná (Manhani \& Lima, 2016) e Ceará (Couto da Silva, 2010). No âmbito da Administração Federal, o trabalho de Vasconcelos (2013) descreveu a informatização do processo de aquisição de insumos estratégicos da área da saúde. A geração de dados e informações de forma sistemática foi outro avanço, em conexão com a implantação dos sistemas informatizados, relatado nas experiências do Rio de Janeiro e Minas Gerais.

Entretanto, os trabalhos mencionados indicam que predominaram as inovações incrementais, sem o enfrentamento das iniciativas de maior complexidade e risco, como é o caso da integração entre as etapas do processo, com a supressão das etapas que não agreguem valor e o aprofundamento da informatização, para ganhar resolutividade na operação das funcionalidades dos sistemas e na integração com outros sistemas. A criação de ferramentas de apoio a etapas do ciclo do suprimento ainda não estruturadas ou precariamente realizadas e as iniciativas de organização da área e de qualificação do seu pessoal pode ser facilitada pelo impulso renovador trazido pelo desenvolvimento e implantação dos sistemas informatizados.

Os portais de compras na internet são uma inovação que ganhou ampla disseminação, em grande medida porque dão visibilidade às licitações públicas e promovem a inclusão da administração pública e seus fornecedores no comércio eletrônico, com potencial de impacto na ampliação da participação, redução de custos de transação e transparência (Ribeiro, 2009). A Administração Federal desenvolveu o seu portal - "Comprasnet" (www.comprasnet.gov.br) -, permitindo que os fornecedores tenham acesso a ferramentas de apoio às compras e a bases de dados interligadas entre todos os órgãos. Estes sistemas oferecem funcionalidades que facilitam a participação dos fornecedores nas licitações públicas, através da publicação de anúncios de licitação na internet e do cadastro online, além da participação direta nos pregões eletrônicos, a partir de qualquer local conectado. 
Estima-se que a ampla maioria dos estados possua portais de compras na internet e que um contingente expressivo realize pregões eletrônicos e algumas transações dos processos de licitação em forma eletrônica, especialmente o cadastro de fornecedores e a divulgação eletrônica dos editais. Pesquisa de Alves \& Souza (2011), realizada em 2010, constatou que 20 dos 27 estados brasileiros mantinham portais de compras, dos quais 12 realizavam pregóes eletrônicos por meio de sistemas próprios. De forma geral, os portais estaduais têm seguido uma configuração predominante que inclui as funcionalidades do pregão e cotação eletrônicos, registro de preços, catálogo de materiais e serviços, publicação das licitações e acompanhamento dos contratos.

A implantação dos portais de compras na internet foi analisada em trabalhos sobre as experiências de São Paulo (Ferreira, Pereira \& Ballesteros, 2016) e Minas Gerais (Hirle et al., 2013). A experiência precursora de São Paulo com a Bolsa Eletrônica de Compras (BEC), criada em 2000, progressivamente evoluiu para se converter em um portal de compras na internet. A BEC introduziu os leilóes reversos para compras públicas no Brasil e uma abrangente padronização das especificações de produtos e serviços. A experiência de Minas Gerais se destaca pelo avanço obtido na abrangência do seu portal de compras, que incorporou as funcionalidades do sistema informatizado de apoio à administração de materiais e serviços.

\subsection{Reestruturação dos formatos de compra e contratação}

A reestruturação dos formatos de compra e contratação se refere às iniciativas de revisão das normas, procedimentos e instrumentos de contratação para atender às características dos produtos e serviços e, ainda, aos perfis de oferta do mercado e de consumo das organizações (Carter \& Narasimhan, 2006). Vários trabalhos apresentaram descrições e análise das experiências estaduais em diversas áreas: A adoção dos cartões de crédito corporativos, o sistema de cotação eletrônica de compras, os acordos de nível de serviço e de melhoria conjunta com terceirizados, a contratação integrada dos serviços de telecomunicação, a negociação direta dos preços de passagens, os sistemas de controle de viagens e novos modelos de abastecimento das frotas.

A implantação dos cartões de crédito para as aquisições de pequeno valor, aprimorando o acompanhamento e controle das despesas, foi inovação com resultados favoráveis, relatados no trabalho de Casagrande, Cestari \& Motta (2009a) sobre o Espírito Santo. No Pará, o sistema de cotação eletrônica de preços foi descrito no trabalho de Barbosa (2015). Trata-se da ferramenta informatizada que possibilita a divulgação de ofertas de compras de pequeno valor e o recebimento de propostas dos fornecedores.

Rodrigues et al. (2013) e Santos, Vimeiro e Rodrigues (2013) descreveram a utilização dos acordos de nível de serviço pelo governo de Minas Gerais, para estipular níveis de desempenho nas contrataçóes, por meio de indicadores. Ferragino \& D'Amico (2010) relataram a padronização do combustível para o abastecimento com álcool nos contratos de locação de veículos e Borges et al. (2010), o caso dos serviços de impressão. A parceria com o fornecedor para a obtenção de melhorias conjuntas é uma inovação praticada nessas experiências.

A adoção de novas estratégias de contratação de serviços tirando proveito das mudanças tecnológicas ocorreu, em especial, na área das telecomunicações. No Espírito Santo, a substituição da telefonia convencional por contratos corporativos de integração de voz, voz sobre Endereço de Protocolo da Internet (IP), imagem e dados levou à redução de custos (Lyrio et al., 2010).

As iniciativas de implantação dos sistemas de gestão da aquisição de passagens para viagens corporativas foram uma frente de inovação com ampla disseminação. São itens de despesa com grande visibilidade perante a opinião pública, como é o caso das passagens aéreas. A adoção de formas inovadoras de negociação dos preços com as empresas de transporte foi quase sempre acompanhada da concentração das compras e da criação de ferramentas, como os sistemas informatizados de compra de passagens e gestão das diárias dos servidores. Em São Paulo (Junqueira, Araújo \& Silva, 2015) e no Piauí (Correia, 2009), a utilização do registro de preços 
permitiu a concentração das compras. No Amazonas (Dos Santos, 2009) e no Espírito Santo (Lyrio et al., $2009,2010)$ foram implantados sistemas informatizados de controle das viagens.

Os modelos de gerenciamento do abastecimento de frota de veículos por meio de cartões ou dispositivos eletrônicos se disseminaram em alguns estados, conforme relatados nos trabalhos sobre Sergipe (Prado, Zylberman \& Andrade, 2008b), Ceará (Santos, 2010) e Minas Gerais (Soares \& Monteiro, 2014). Em Sergipe, foi implantado o controle eletrônico do consumo da frota por meio de dispositivo instalado diretamente em cada veículo. O Ceará implantou um sistema de gestão para o consumo de combustível baseado na descentralização das compras, utilizando cartão magnético e ferramenta informatizada de controle. $\mathrm{O}$ modelo de Minas Gerais utiliza rede própria de abastecimento, seguindo opção adotada com base na comparação de custos em relação à compra em postos comerciais credenciados.

\subsection{Terceirização}

A terceirização é frequentemente objeto de controvérsia no Brasil, embora a descentralização de atividades para a contratação no mercado seja expressamente prevista como forma de descentralização desde a publicação da lei da reforma administrativa de 1967, ainda em vigência (Decreto-lei nº 200), que recomenda a "execução indireta", mediante contrato, das atividades que a iniciativa privada esteja apta a desempenhar (veja art. $10, \$ 7^{\circ}$ ). A sua disseminação internacional recente está associada às políticas de redução de custos e descentralização e tem sido adotada a despeito das diferenças de cultura, estrutura e organização entre as administrações públicas nacionais, conforme levantamento da OCDE (2010). A contratação de serviços exige a internalização de capacidades técnicas específicas, que incluem metodologias e instrumentos para apoiar a decisão pela terceirização, a especificação dos serviços e o seu monitoramento (Ancarani, 2009). Implementada como solução imediatista ou desvinculada de uma visão estratégica, a terceirização pode gerar problemas de coordenação, instrumentalização dos contratos e perda de controle e accountability (Kirkpatrick \& Lucio, 1996).

Os trabalhos de Teixeira, Martelanc e Prado Filho (2009) e de Dantas (2012) buscaram desfazer preconceitos e simplificações para argumentar em favor da utilização da terceirização, desde que atendidos critérios de conveniência e economicidade e somente quando a administração pública dispor de padrões adequados para definir formatos de contratação, preços de referência e avaliação da prestação dos serviços. $\mathrm{O}$ trabalho de Teixeira et al. (2010) recomendou a aplicação dos critérios e parâmetros da teoria dos contratos para fundamentar as definições estratégicas da administração pública em relação à alternativa da produção direta (verticalização) ou contratação. Dantas (op. cit.) discutiu os riscos jurídicos da terceirização utilizada de forma descuidada, como no caso da contratação para reposição de força de trabalho e não para prestação de serviços.

A criação de um órgão de caráter técnico para estabelecer os padrões e a supervisão das contratações foi uma importante inovação em São Paulo (Ferragino \& D’Amico, 2010). O trabalho de Agune e Ferragino (2009) analisou a experiência da implantação das metodologias para a decisão pela terceirização dos serviços de prestação continuada. A terceirização da frota foi adotada em Minas Gerais, com base na análise sistemática das alternativas de compra ou locação dos veículos (Brito, Duarte \& Soares, 2014). A inovação na terceirização para a contratação de serviços de gestão de prestadores "quarterizados" foi implantada, nesse mesmo estado, para os serviços de manutenção da frota de veículos de uso corporativo pela administração pública (Soares \& Moura, 2014). No Tocantins, foram terceirizados os serviços de logística para a gestão do fluxo de compra, armazenagem e distribuição de medicamentos e materiais hospitalares (Coelho, 2010). 


\subsection{Metodologias e ferramentas de gestão das compras}

As inovações em metodologias e ferramentas de gestão das compras públicas incluem o planejamento das compras, padronização dos materiais e serviços, estimação dos preços de referência das licitações, controle de custos, gestão dos contratos e avaliação dos fornecedores. O planejamento das compras e contratações e a consequente agregação das demandas e aproveitamento dos ganhos de escala foram avanços que se realizaram a partir da assimilação da abordagem estratégica das compras públicas. Em Minas Gerais foram criadas agendas de compras e coordenação das licitações (Silva, Rodrigues \& Anjos, 2009; Rodrigues \& Marques, 2010) buscando o seu alinhamento com os objetivos e metas do planejamento governamental (Vilhena \& Hirle, 2013). Na Bahia, a criação de catálogos para a padronização dos materiais foi relatada no trabalho de Gomes (2010).

As metodologias para estimação dos preços de referência das contratações vieram substituir a simples coleta informal de cotações. No Espírito Santo foi introduzida uma metodologia baseada em pesquisa de mercado (Cestari, Casagrande \& Motta, 2008; Casagrande, Cestari \& Motta, 2009b). A estimação a partir dos preços diretamente registrados nas transações comerciais é a metodologia implementada no Rio Grande do Sul (Leal \& Mahler, 2013) e no Amazonas (Silva, 2016). O trabalho de Ferreira (2013) relatou o desenvolvimento de ferramentas de inteligência computacional para o tratamento estatístico dos preços de referência das licitações e para a produção de relatórios gerenciais, em São Paulo. Um sistema de controle de custos capaz de identificar oportunidades de economia, abrangendo várias etapas do ciclo do suprimento, foi implantado em Pernambuco (Sales, 2014).

O trabalho de Drumond et al. (2013) relatou a introdução, em Minas Gerais, de mecanismos sistemáticos de levantamento de informações sobre os fornecedores que possibilita a identificação de gargalos de suprimento e a melhoria do seu relacionamento com o Estado. A metodologia de estimação dos custos dos projetos e atividades foi objeto do trabalho de Silveira (2010), que analisou a experiência do Banco Central com a implantação do sistema de custo por atividade (ABC - Activity-Based Costing). O trabalho de Morais, Silva \& Cardoso (2009) e de Cardoso et al. (2011) relataram a experiência do controle dos custos de mão-deobra dos contratos de terceirização no Rio Grande do Norte, que inibiu o pagamento de propinas por meio da especificação precisa das despesas em planilhas.

\subsection{Uso do poder de compra}

As políticas de uso do poder de compra do Estado visam alinhar as compras públicas a objetivos socioeconômicos. Dessa forma, as compras e contratações públicas podem ser incorporadas ao desenho de políticas de alcance transversal, como instrumento para alcançar resultados e impactos mais amplos, que sejam de interesse público (Grandia \& Meehan, 2017). Há uma diversidade de métodos e instrumentos, sendo alguns dos mais conhecidos o estabelecimento de regras e requisitos para a seleção de fornecedores e propostas e para o cumprimento de contratos (Arrowsmith, 2010). As políticas desta natureza, no Brasil, têm sido criadas em duas frentes, de forma combinada ou não: A primeira se volta para o uso do poder de compra no fomento das micro e pequenas empresas e no desenvolvimento local. A segunda mira a sustentabilidade ambiental das compras e contratações e, de forma derivada, do consumo e do descarte de bens e insumos.

As políticas em favor das micro e pequenas empresas se disseminaram nos estados, impulsionadas pela aprovação, em 2006, do Estatuto da Microempresa. As regras de preferência foram o principal instrumento dessas políticas, inserido no arcabouço legal das licitações, impondo aos órgãos a necessidade de lidar com a aplicação conjunta dos diversos critérios e parâmetros de seleção de propostas (Arantes, 2015). As regras passaram por sucessivas ampliações em seu alcance, primeiro, com a criação do "empate ficto" e a preferência para as microempresas e empresas de pequeno porte, em 2006. Em 2007, foi regulamentada a preferência para 
bens e serviços de informática e automação e em 2010, as margens de preferência para produtos e serviços com tecnologia nacional.

Em Pernambuco (Rocha et al., 2013) e no Rio Grande do Sul (Dasso Jr., 2013; Torres, Mayer \& Lunardi, 2013) foram criados programas governamentais de apoio à participação das micro e pequenas empresas nas compras públicas e em Sergipe foi aprovada uma lei estadual com este propósito (Teles et al., 2009). O Programa "Fornecer", no Rio Grande do Sul, orienta as empresas locais a obterem habilitação para participar de pregões presenciais para compra de alimentos de consumo da própria administração estadual, em penitenciárias, escolas e hospitais. Em Minas Gerais a ampliação da base de fornecedores foi objeto de uma iniciativa que integrou a abertura de empresas, nas juntas comerciais, ao seu cadastramento automático como fornecedor (Souza et al., 2013).

Os compromissos internacionais assumidos pelo país e a iniciativa coordenadora, no âmbito da administração federal, levaram à criação de uma política de sustentabilidade ambiental com alcance transversal (Barki \& Gonçalves-Dias, 2014). As compras sustentáveis têm sido incorporadas às agendas da administração federal e de estados como São Paulo (D’Amico \& Agune, 2009; D’Amico, 2010).

\subsection{Ciclo do suprimento}

A visão das compras e contratações como uma função da administração pública está baseada na definição sistêmica dos seus componentes e relações como processos interdependentes que perpassam o conjunto dos órgãos e têm como eixo fundamental o suprimento dos bens e serviços (Thai, 2001; 2009). O ciclo do suprimento é constituído pelas etapas de identificação de necessidades, especificação do produto e do fornecimento, divulgação ao mercado e análise e seleção de propostas, escolha dos fornecedores, negociação de preços e de condições, elaboração e celebração do contrato, recebimento, inspeção e armazenamento do bem, controle de estoques e reposição. No caso de contratos de prestação de serviços, inclui as etapas de gestão dos contratos. O processo do suprimento se completa, necessariamente, com sua articulação externa, junto ao mercado dos fornecedores, na forma dos processos de compra ou contratação de bens e serviços (Tridapalli, Fernandes \& Machado, 2011).

A estruturação do ciclo do suprimento como processos integrados, capazes de atender de forma satisfatória às necessidades de gestão da administração pública com eficiência e regularidade, é um tema de agenda governamental no Brasil ainda não resolvido. O trabalho de Teixeira et al. (2011) criticou a prevalência no Brasil de visões limitadas e segmentadas das compras e contratações públicas. A função suprimento vem sendo alçada ao nível estratégico das organizações, inclusive pela formulação de um planejamento de compras e contratações que seja capaz de estabelecer objetivos e estratégias, ao longo de toda a cadeia de valor, considerando a contribuição de cada elo à geração de valor pela organização (Cooper, Lambert \& Pagh, 1997). Esse planejamento pode incluir as decisões sobre o que produzir internamente, comprar ou terceirizar e o tratamento dos fornecedores como parceiros em estratégias para a geração de valor (Prado Filho et al., 2016). O trabalho de Ferrer (2013) defendeu um modelo abrangente e integrado, adotando a perspectiva do ciclo do suprimento.

2.8.Centralização das compras

A centralização das compras é uma tendência evidenciada pela recente criação das agências de compras, nos países europeus, como modelo voltado para o aproveitamento de ganhos de escala e a condução qualificada das contratações ou aquisições de maior complexidade (Dimitri et al., 2006). Ao mesmo tempo, proliferam as iniciativas de atuação colaborativa em torno do compartilhamento das compras entre organizações públicas, sob múltiplos formatos e circunstâncias (Walker et al., 2007), mas a descentralização por meio da delegação de atividades e rotinas, apoiada em sistemas de informação, é também uma tendência que vem se contrapor ao modelo tradicional dos departamentos de compra (Thai, 2001). Na verdade, levantamentos 
empíricos indicam a predominância de modelos mistos que combinam componentes de centralização e descentralização, em diferentes graus e formatos (McCue \& Pitzer, 2000).

No caso brasileiro, a fragilidade política e institucional das compras levou à descontinuação do modelo de centralização do período Vargas, adotado entre os anos 1930 e 1945, deixando como legado uma situação de fragmentação e autonomia exagerada dos órgãos ao longo do período subsequente (Fernandes, 2015). Essa situação tem sido revertida nos últimos anos com o fortalecimento dos órgãos centrais responsáveis pela supervisão e normatização das compras e com as iniciativas de centralização, a exemplo da criação da Central de Compras e Contratações do Governo Federal, e de compartilhamento de compras. Em Minas Gerais, a contratação centralizada dos serviços de uso comum foi implantada por ocasião da instalação da Cidade Administrativa, sede do governo estadual, inaugurada em 2010 (Rodrigues et al., 2013). A criação de uma unidade especificamente voltada para a gestão e fiscalização dos serviços reuniu as equipes responsáveis pela contratação e pela fiscalização, que passou a se apoiar em acordos de nível de serviço.

No Ceará, a centralização foi solução adotada para a redução de custos dos hospitais da rede pública, reforçando a gestão dos estoques num contexto de pressão fiscal (Souza \& Freitas, 2016). Há registros sobre as experiências de centralização das compras em Sergipe, Pernambuco e Pará, implementadas em conexão com a regulamentação do registro de preços (veja seção 2.1), mas não é possível identificar experiências mais avançadas de fortalecimento da estrutura organizacional das compras e contratações, que deveria ser acompanhada da criação de órgãos ou instâncias de centralização ou coordenação. Além disso, experiências como a do Ceará sugerem que não se partiu propriamente de um modelo descentralizado ao qual se contrapusesse a centralização, mas da debilidade do arcabouço de normas e, sobretudo, dos padrões e parâmetros para a gestão e controle das licitações.

\subsection{Institucionalização da função compras e contratações}

Os fatores de ordem institucional são importantes para analisar a consolidação das compras e contratações públicas sob uma perspectiva mais abrangente, como função da administração pública cuja construção exige o equacionamento de requisitos relacionados com a estrutura organizacional e a inserção dessa área, em termos de sua identidade, espaço de atuação e jurisdição, leis, normas, políticas e procedimentos (Hunja, 2003). A organização da área na administração federal foi analisada por Fernandes (2014), que identificou uma crônica debilidade em sua trajetória, caracterizada pela ausência de uma estrutura organizacional própria e de uma delimitação precisa e abrangente do seu espaço de atuação.

O trabalho de Teixeira, Silva e Salomão (2014) explorou alguns desses aspectos, no caso das administrações públicas estaduais e salienta como particularmente problemática a inexistência de quadros de pessoal especializado que, organizado como carreira ou em outros formatos, fortaleça a identidade profissional, a expertise técnica e a construção de uma identidade pelos funcionários em atuação nestas atividades. Além disso, aponta Minas Gerais como o caso mais desenvolvido de integração entre os componentes de uma política de gestão do suprimento. Avanços teriam ocorrido também em São Paulo e Rio de Janeiro, com a criação de ferramentas informatizadas; no Rio Grande do Sul, com a centralização das compras, acompanhada de mudanças nos processos e criação de ferramentas de tratamento dos dados, e em Goiás, com o início do desenvolvimento de um sistema de logística e gestão de suprimentos.

\section{Conclusões: AVAnÇOS, Dificuldades E PERSPECtivas}

A área de compras e contratações públicas revelou-se inegavelmente dinâmica e inovadora quando observamos sua trajetória recente, retratada na amostra de trabalhos selecionados. Entretanto, não há rupturas ou inflexões acentuadas na trajetória, mas uma progressiva disseminação de inovações do governo 
federal para os estados e municípios e, mais recentemente, entre os estados e municípios. Essa disseminação parece ter sido impulsionada pelo intercâmbio direto de experiências, pela cooperação em moldes mais formais, pelo trabalho de consultores e por organismos internacionais que passaram a se interessar pela modernização e convergência entre os modelos de compras e contratações públicas.

Duas inovações no arcabouço legal e normativo, criadas na Administração Federal, mudaram as regras de contratação e de seleção e negociação de propostas nas licitações: O pregão e o registro de preços. Elas foram as matrizes da proliferação de inovações que em muitos casos se combinaram entre si e com outras inovações, aprofundando e ampliando seus impactos, por exemplo, pela utilização do registro de preços para padronizar as compras ou para concentrar e centralizar as licitações, ou pela disseminação do pregão como instrumento da inclusão de micro e pequenas empresas como fornecedores públicos.

Outra inovação igualmente importante na trajetória foi a criação dos portais de compras na internet, que teve no "Comprasnet" e no sistema eletrônico de apoio às licitações da administração federal dois importantes vetores da disseminação do comércio eletrônico na administração pública, alcançando estados e municípios através de convênios e cooperação. A ampla maioria dos estados atualmente mantém portais de compras e muitos realizam pregões online e diversas transações importantes dos processos de licitação em forma eletrônica, como o cadastro de fornecedores e a divulgação dos editais na internet.

Os avanços que se pode constatar nas compras e contratações públicas demonstram que a agenda dessa área se diversificou, abrindo novas frentes para além das mudanças legais e normativas nas regras de licitação. As principais tendências de inovação e mudança foram, em primeiro lugar, a criação de novos formatos de contratação, a partir de um conhecimento melhor da administração pública sobre os produtos e serviços no mercado e as suas necessidades e perfil de consumo. Enquadram-se nessa tendência os cartões corporativos para suprimento de fundos ou abastecimento de frotas, os acordos de nível de serviços com os terceirizados, a contratação de serviços compartilhados entre vários órgãos, a negociação direta dos preços de passagens e a contratação integrada dos serviços de telecomunicação.

Uma segunda tendência foram as metodologias e ferramentas de gestão das compras, que se desenvolveram apoiadas no aproveitamento dos potenciais da tecnologia da informação. Exemplos desse tipo de inovação foram as experiências de informatização de várias etapas do fluxo processual das licitações dentro dos órgãos, os sistemas e metodologias de planejamento das compras, a padronização dos materiais e serviços, a estimação dos preços de referência, a gestão de contratos, a avaliação de fornecedores e a integração das licitações ao orçamento, além da construção de indicadores gerenciais sobre a área.

O uso do poder de compra do Estado foi uma terceira tendência de mudança e inovação que, neste caso, voltou-se para a incorporação de objetivos socioeconômicos a esta área, seja por meio do fomento a micro e pequenas empresas e ao desenvolvimento local, seja pela introdução de parâmetros de sustentabilidade ambiental para as compras e contratações e para o consumo de bens e serviços pela administração pública. Várias experiências relatadas nos trabalhos analisados demonstram que esses temas têm encontrado respaldo entre os governos nos três níveis, alinhando as iniciativas da área a uma agenda internacional com grande visibilidade pública.

Ressalte-se que os avanços registrados ainda permanecem limitados em sua abrangência e profundidade porque falta estruturar o ciclo do suprimento, ou seja, o conjunto das etapas que compõem, no seu conjunto, a gestão das compras e contratações, desde a identificação das necessidades até o controle dos estoques e a avaliação da prestação dos serviços terceirizados. Muitas das etapas estão ainda em uma situação na qual a sua estruturação é ainda item de agenda a ser enfrentado pelas administrações públicas, o que exigirá a capacitação de pessoal, a assimilação de metodologias e expertise técnica e a implantação de sistemas e ferramentas de gestão.

De resto, a necessidade de fortalecimento institucional da área é questão central que se depreende do exame de muitos dos trabalhos, quando atentamos para as dificuldades e limitações relatadas pelos autores, a maioria practitioners que vivem a realidade cotidiana da gestão pública. As compras e contratações públicas devem 
ser encaradas como uma função da administração pública cuja construção exige o equacionamento de um conjunto de requisitos relacionados com a estrutura organizacional e a inserção da área, em termos de sua identidade, do espaço de atuação e jurisdição, das leis, das normas, das políticas e dos procedimentos.

O levantamento realizado permite inferir indicações de temas para pesquisas futuras que contribuam para a solução de problemas, o desenvolvimento de modelos e instrumentos e o aprofundamento do conhecimento sobre as compras públicas. Fundamentalmente, sugere-se a realização de levantamentos bibliométricos da produção brasileira mais abrangentes, que analisem de forma integrada e com a devida categorização os trabalhos dos practitioners e os de origem acadêmica. Além disso, é necessário incorporar a produção representada pelos trabalhos dos programas de pós-graduação. O levantamento de dados sobre os estados e municípios, que permanecem em larga medida pouco conhecidos, é outra linha de trabalho premente a ser viabilizada tanto para subsidiar as políticas governamentais como também para servir à pesquisa empírica, particularmente os estudos comparados.

A prospecção, análise e proposição de modelos de gestão das compras públicas e da cadeia de suprimentos que sejam adequados às especificidades do setor público é outra oportuna linha de pesquisa aplicada que emerge como oportunidade, a partir dos resultados do balanço apresentado nesse artigo. Da mesma forma, parece necessária e oportuna a pesquisa sobre a terceirização e seus resultados e a aplicação de abordagens institucionais e organizacionais que se voltem para a área de compras e contratações, na forma de estudos de caso e pesquisa comparada.

\section{REFERÊNCIAS}

Agune, R. M. \& Ferragino, H. J. (2009, maio) Construindo um modelo para terceirização de um serviço. Congresso CONSAD de Gestão Pública, Brasília, DF, Brasil, 2.

Alves, T. R. \& Souza, C. A. (2011). Compras eletrônicas governamentais: uma avaliação dos sites de e-procurement dos governos estaduais. Revista Eletrônica de Sistemas de Informação 10 (1).

Amorim, P. C. L. (2016, julho). Os efeitos da modalidade pregão na execução dos contratos continuados: um estudo de caso. Congresso CONSAD de Gestão Pública, Brasília, DF, Brasil, 9.

Ancarani, A. Service sourcing (2009). In Thai, K. V. (ed.) International Handbook of Public Procurement, p. 187-210. Boca Raton, London, N. York: CRC Press.

Arantes, R. S. (2015, maio). A aplicação conjunta das preferências sobre o preço nas compras governamentais do governo federal. Congresso CONSAD de Gestão Pública, Brasília, DF, Brasil, 8.

Araújo, R. S. R. \& Gomes, C. S. (2010, março). Análise comparativa da "vantajosidade" entre compras governamentais centralizadas e descentralizadas. Congresso CONSAD de Gestão Pública, Brasília, DF, Brasil, 3.

Arrowsmith, S. (2010). Horizontal policies in public procurement: a taxonomy, Journal of Public Procurement, 10 (2), 149-186.

Arrowsmith, S. \& Davies, A. (eds.) (1998). Public Procurement: Global Revolution. London: Kluwer Law International.

Arrowsmith, S. \& Trybus, M. (eds.) (2003). Public Procurement: The Continuing Revolution. London: Kluwer Law International.

Barbosa, E. F. (2015, maio). O governo eletrônico e a gestão da cadeia de suprimentos do setor público. Congresso CONSAD de Gestão Pública. Brasília, DF, 8.

Barki, T. V. \& Gonçalves-Dias, S. L. F. (2014, março). Licitações sustentáveis no Brasil: aspectos jurídicos e de gestão pública. Congresso CONSAD de Gestão Pública, Brasília, DF, Brasil, 7.

Bezerra, S. Q., Portela, A. M. C., Lopes, J. H. P. \& Coelho Bezerra, F. J. (2010, março). O Sistema de Gestão de Registro de Preços do Governo do Estado do Ceará. Congresso CONSAD de Gestão Pública, Brasília, DF, 3.

Borges, B., Guido, R., Ferragino, H. J. \& Teixeira, H. J. (2010, março). Melhoria contínua na contratação de serviços terceirizados: o caso da impressão corporativa. Congresso CONSAD de Gestão Pública, Brasília, DF, 3. 
Brito, F. S., Duarte, J. M. \& Soares, M. E. S. (2014, março). A terceirização da frota de veículos oficiais: a experiência Mineira com a locação de veículos administrativos, operacionais e de representação. Congresso CONSAD de Gestão Pública, Brasília, DF, 7.

Campos, L. C. M. (2008, novembro). Compras governamentais: mapeamento preliminar de estudos na área de administração pública e das principais questões de pesquisa. Encontro de Administração Pública e Governança (ENAPG), Salvador, BA.

Cardoso, R. G., Guido, R. V. C., Medeiros, I. C. N. \& Lippi, M. F. (2011, maio). Renegociação dos contratos de serviços terceirizados pelo Governo do Estado do Rio Grande do Norte. Congresso CONSAD de Gestão Pública, Brasília, DF, 4.

Carter, J. R. \& Narasimhan, R. (2006). Purchasing and Supply Management: Future Directions and Trends. Journal of Supply Chain Management 32 (4), 2-12.

Casagrande, M. L., Cestari, A. N. \& Motta, A. P. P. D. (2009a, maio). Controle do gasto público desde as pequenas compras". Congresso CONSAD de Gestão Pública, Brasília, DF, 2.

Casagrande, M. L., Cestari, A. N. \& Motta, A. P. P. D. (2009b, maio). Preços referenciais: economia, rapidez e qualidade nas compras governamentais. Congresso CONSAD de Gestão Pública, Brasília, DF, 2.

Cestari, A. N., Casagrande, M. L. \& Motta, A. P. P. D. (2008, maio). Celeridade nos processos de aquisições públicas: fim dos orçamentos. Congresso CONSAD de Gestão Pública, Brasília, DF, 1.

Coelho, E. P. F. (2010, março). Logística de dispensação na rede de saúde pública. Congresso CONSAD de Gestão Pública, Brasília, DF, 3.

Cooper, M. C., Lambert, D. M. \& Pagh, J. D. (1997). Supply chain management: more than a new name for logistic, The International Journal of Logistics Management 8 (1), 1-14.

Correia, F. S. C. B. (2009, maio). Criação e importância da central de compras de passagens aéreas na Secretaria da Administração do Governo do Piauí. Congresso CONSAD de Gestão Pública, Brasília, DF, 2.

Couto da Silva, S. J. (2016, julho). E-recebimento - Sistema de Recebimento de Materiais do Amazonas. Congresso CONSAD de Gestão Pública, Brasília, DF, 9.

Croom, S. \& Brandon-Jones, A. (2005). Key issues in e-procurement: procurement implementation and operation in the public sector. Journal of Public Procurement, 5 (3), 367-387.

D’Amico, V. (2010, março). Desenvolvimento sustentável: poder de compra. Congresso CONSAD de Gestão Pública, Brasília, DF, 3.

D’Amico, V. \& Agune, R. M. (2009, maio). Contratações públicas sustentáveis - a experiência do governo de São Paulo. Congresso CONSAD de Gestão Pública, Brasília, DF, 2.

Dantas, F. A. B. (2012, junho). A terceirização como instrumento de gestão na administração pública. Congresso CONSAD de Gestão Pública, Brasília, DF, 5.

Dasso Jr., A. E. (2013, abril). Programa Fornecer/RS: utilizando compras públicas para fazer políticas públicas de distribuição de renda. Congresso CONSAD de Gestão Pública, Brasília, DF, 6.

Dezolt, A. L. P., Tachlian, E., Santos, M. S., Harper, L. \& Barbosa, G. P. (eds.) (2016). Compras públicas estaduais Boas práticas brasileiras. Brasília: Conselho Nacional de Secretários de Estado de Administração (CONSAD).

Dezolt, A. L. P. \& Barbosa, G. P. Diagnóstico das compras públicas estaduais 2015. In A. L. P. Dezolt, E. Tachlian, M. S. Santos, L. Harper \& G. P. Barbosa (eds.) (2016). Compras públicas estaduais - Boas práticas brasileiras. Brasília: Conselho Nacional de Secretários de Estado de Administração (CONSAD), p. 15-34.

Dimitri, N., Dini, F., Piga, G. \& Zanza, Matteo. When should procurement be centralized? (2006) In N. Dimitri, G. Piga \& G. Spagnolo (eds.) Handbook of Procurement. Cambridge: Cambridge University Press, p. 47-81.

Dimitri, N., Piga, G. \& Spagnolo, G. Introduction (2006). In Idem (eds.). Handbook of Procurement. Cambridge: Cambridge University Press.

Dos Santos, S. A. C. (2009, maio). Sistema de Controle de Diárias e Passagens. Congresso CONSAD de Gestão Pública, Brasília, DF, 2. 
Drumond, L. V. S., Zolini, T. M., Maciel, L. L. B. \& Diniz, L. C. (2013, abril). Instituição de metodologia para a avaliação do desempenho de fornecedores pelos órgãos e entidades do poder executivo do estado de Minas Gerais: desenvolvimento, implantação e benefícios. Congresso CONSAD de Gestão Pública, Brasília, DF, 6.

Fernandes, C. C. C. (2014, março). A organização da área de compras e contratações públicas na administração pública federal brasileira - o elo frágil. Congresso CONSAD de Gestão Pública, Brasília, DF, 7.

Fernandes, C. C. C. (2015, maio). A centralização das compras na administração federal - lições da história. Congresso CONSAD de Gestão Pública, Brasília, DF, 8.

Fernandes, C. C. C. (2016, julho). A experiência do pregão nas compras públicas: oportunidade e inovação. Congresso CONSAD de Gestão Pública, Brasília, DF, 9.

Ferragino, H. J. \& D`Amico, V. (2010, março). Modernização de modelo de negócios: locação de veículos e gerenciamento de combustível. Congresso CONSAD de Gestão Pública, Brasília, DF, 3.

Ferreira, M. F. A. (2013, abril). Inteligência em compras: o novo desafio da bolsa eletrônica de compras de São Paulo. Congresso CONSAD de Gestão Pública, Brasília, DF, 6.

Ferreira, M. F. A., Pereira, V. E. \& Ballesteros, A. S. (2016, junho). A Contribuição da Bolsa Eletrônica de Compras do Governo do Estado de São Paulo para melhoria da gestão pública. Congresso CONSAD de Gestão Pública, Brasília, DF, 9.

Ferrer, F. (2013, abril). Os desafios do setor público para construir um novo modelo de compras. Congresso CONSAD de Gestão Pública, Brasília, DF, 6.

Fiuza, E. P. S. \& Medeiros, B. A. (2014). A agenda perdida das compras públicas: rumo a uma reforma abrangente da lei de licitações e do arcabouço institucional. Texto para discussão - Instituto de Pesquisa Econômica Aplicada, 1990.

Gomes, A. J. S. (2010, março). Padronização das Descrições de Materiais (PDM). Congresso CONSAD de Gestão Pública, Brasília, DF, 3.

Grandia, J. \& Meehan, J. (2017). Public procurement as a policy tool: using procurement to reach desired outcomes in society. International Journal of Public Sector Management, 30 (4), 302-309.

Hirle, A. L. C., Duarte, J. M., Drumond, L. V. S. \& Teixeira, R. S. V. (2013, abril). Inovaçóes no Portal de compras MG: soluções para o suporte, controle e gestão dos processos de compras públicas em Minas Gerais. Congresso CONSAD de Gestão Pública, Brasília, DF, 6.

Hirle, A. L. C., Drumond, L. V. S., Soares, M. E. S. \& Souza, W. K. A. (2015, maio). Indicadores: reflexões sobre a sua contribuição para o alcance de resultados em políticas de gestão de suprimentos de Minas Gerais. Congresso CONSAD de Gestão Pública, Brasília, DF, 8.

Hunja, R. R. (2003). Obstacles to Public Procurement Reform in Developing Countries. In S. Arrowsmith, S. \& M. Trybus (eds.). Public Procurement: The Continuing Revolution. London: Kluwer Law International.

Junqueira, M. O., Araujo, D. G. \& Silva, J. O. (2015, maio). Desafios da centralização das compras públicas: o caso das passagens aéreas do Estado de São Paulo. Congresso CONSAD de Gestão Pública, Brasília, DF, 8.

Kirkpatrick, I. \& Lucio, M. (1996). The contract state and the future of public management. Public Administration, 74 (Spring), 1-8.

Knight, L., Harland, C., Telgen, J., Thai, K. V., Callender, G. \& Mcken, K. (ed) (2007). Public Procurement: International cases and commentary. London/N. York: Routledge.

Leal, J. P. \& Mahler, P. R. (2013, abril). Atenção com o preço justo de mercado: economizando nas compras públicas com o uso da nota fiscal eletrônica. Congresso CONSAD de Gestão Pública, Brasília, DF, 6.

Lyrio, A. B., Perez, C. S., Gomes, H. N., Fernandes, S. C. R. \& Goggi, W. (2009, maio). Celeridade, economia e controle na aquisição de passagens aéreas e diárias por meio de Sistema Informatizado de Gerenciamento. Congresso CONSAD de Gestão Pública, Brasília, DF, 2.

Lyrio, A. B., Perez, C. S., Gomes, H. N., Fernandes, S. C. R. \& Goggi, W. (2010, março). Gestão inovadora de viagens com celeridade, economia e controle nos gastos de passagens aéreas e diárias. Congresso CONSAD de Gestão Pública, Brasília, DF, 3. 
Lyrio, A. B., Nunes, G. S., Ribeiro, J. H. \& Gomes, H. N. (2010, março). Tecnologia da informação e ferramentas aplicadas às compras. Congresso CONSAD de Gestão Pública, Brasília, DF, 3

MacManus, S. A. (1996). Designing and Managing the Procurement Process. In James L. Perry (ed.) Handbook of Public Administration. San Francisco: Jossey-Bass.

MacManus, S.A. (2002). Understanding the incremental nature of e-procurement implementation at the state and local levels. Journal of Public Procurement, 2 (1), 5-28.

McCue, C. P. \& Pitzer, J. T. (2000). Centralized vs. Decentralized purchasing: current trends in government procurement practices. Journal of Public Budgeting, Accounting \& Financial Management, 12 (3), 400-420.

McCue, C. \& Roman, A. V. (2012). E-Procurement: myth or reality? Journal of Public Procurement, 12 (2), 221-248.

Manhani Jr., M. \& Lima, M. (2016, julho). Avanços e perspectivas nos processos de compra e contratação de serviços realizados pela Secretaria de Estado da Administração e da Previdência, por intermédio do Departamento de Administração do Material. Congresso CONSAD de Gestão Pública, Brasília, DF, 9.

Marini, C. (2008). Gestão das compras governamentais: perspectivas para a consolidação dos avanços. In P. C. Medeiros (ed). Avanços e Perspectivas da Gestão Pública nos Estados. São Paulo: Qualitymark.

Moon, M. J. (2005). E-procurement management in state governments: diffusion of e-procurement practices and its determinants. Journal of Public Procurement, 5 (1), 54-72.

Morais, M. M., Silva, M. R. \& Cardoso, R. G. (2009, maio). Elementos de composição de custos de terceirização de mão de obra nos serviços de limpeza e conservação. Congresso CONSAD de Gestão Pública, Brasília, DF, 2.

Nagle, James F. (1999). A history of Government contracting. Washington, DC: The George Washington University Press, Government Contract Program.

Neef, D. (2001). E-Procurement: From Strategy to Implementation, Upper Saddle River- NJ: Prentice Hall.

Negrão, I. A. M. \& Matos, T. F. (2016, junho). Sistema de registro de preços nas compras governamentais do Estado do Pará. Congresso CONSAD de Gestão Pública, Brasília, DF, 9

OCDE (2010). Public Administration after "New Public Management", Organização para a Cooperação e Desenvolvimento Econômico.

Oliveira, H. A., Pessoa Neto, J. A. \& Torres, R. B. (2013, abril). Regime diferenciado de contratações públicas - RDC: a experiência da INFRAERO. Congresso CONSAD de Gestão Pública, Brasília, DF, 6.

Oliveira, H. A., Pessoa Neto, J. A. \& Torres, R. B. (2015, maio). Regime diferenciado de contratações públicas (RDC) e a eficácia do regime de contratação integrada das obras públicas: experiência INFRAERO. Congresso CONSAD de Gestão Pública, Brasília, DF, 8.

Oshiro, W. H., Renda dos Santos, L.M. \& Jesus-Lopes, J.C. (2016). Compras Públicas: Levantamento Bibliométrico da Produção Científica do Portal de Periódicos CAPES/MEC, entre 1995 e 2015. Congresso Nacional de Mestrados Profissionais em Administração Pública, Curitiba, PR, 1.

Pacano, M., Pinto, M. T. M., Nunes, F. A. S. (2010, março). Sistema Integrado de Gestão de Aquisições (SIGA): transformação na área de suprimentos e logística no Governo do Rio de Janeiro. Congresso CONSAD de Gestão Pública, Brasília, DF, 3.

Patrucco, A. S., Luzzini, D. \& Ronchi, S. (2017). Research perspectives on public procurement: Content analysis of 14 years of publications in the Journal of Public Procurement, Journal of Public Procurement, 17 (2), 229-269.

Prado, J. A. T., Zylberman, M. \& Andrade, G. (2008a, maio). Da previsão legal à aplicabilidade prática: a contratação via Sistema de Registro de Preço adotada pelo Governo do Estado de Sergipe. Congresso CONSAD de Gestão Pública, Brasília, DF, 1.

Prado, J. A. T., Zylberman, M. \& Andrade, G. (2008b, maio). O processo de contratação centralizada utilizado pelo Governo do Estado de Sergipe: a experiência de gestão do abastecimento da frota de veículos. Congresso CONSAD de Gestão Pública, Brasília, DF, 1.

Prado, V. J. N. (2014). A Produção Científica Relacionada ao Tema Compras Públicas nos anais do EnANPAD e Congresso USP de Controladoria e Contabilidade: Análise Bibliométrica no período de 2008-2013. Monografia de bacharelado, Universidade de Brasília, Brasília, DF. 
Prado Filho, J. L. (2008, maio). O uso do poder de compra do governo estadual e municipal enquanto indutor do desenvolvimento econômico e social: o caso de Sergipe. Congresso CONSAD de Gestão Pública, Brasília, DF, 1 .

Prado Filho, L. P. C., Guido, R. V. C., Teixeira, H. J., \& Ribeiro, E. E. M. D. (2016, julho). Reduzindo os Custos das Contratações de Serviços Terceirizados com Planejamento, Inovação e Produtividade. Congresso CONSAD de Gestão Pública, Brasília, DF, 9.

Ribeiro, M. M. (2009, maio). Como os estados brasileiros promovem a transparência nos portais de compras eletrônicas? Congresso CONSAD de Gestão Pública, Brasília, DF, 2.

Rocha, D. T. M., Ferreira, L. C., Rodrigues, R. A. \& Soares, M. F. (2013, abril). Desenvolvimento de política de compras a microempresas, empresas de pequeno porte e microempreendedores individuais no Estado de Pernambuco." Congresso CONSAD de Gestão Pública, Brasília, DF, 6.

Rodrigues, G. \& Marques, R. L. (2010, março). A experiência e os resultados da primeira onda do planejamento de consumo na SES/MG. Congresso CONSAD de Gestão Pública, Brasília, DF, 3.

Rodrigues, G., Duarte, K. K. D., Tomich, M. V. S. \& Coelho, R. R. (2013, abril). Acordo de nível de serviço e eficiência na gestão contratual: O caso da Cidade Administrativa. Congresso CONSAD de Gestão Pública, Brasília, DF, 6.

Sales, L. S. (2014, março). Mecanismos de controle no ciclo das compras governamentais e oportunidades de racionalização do gasto público: a experiência da Secretaria da Administração do Estado de Pernambuco. Congresso CONSAD de Gestão Pública, Brasília, DF, 3.

Santos, G. H. C., Vimeiro, J. V. \& Rodrigues, M. I. A. (2013, abril). Acordo de nível de serviço e eficiência administrativa. Congresso CONSAD de Gestão Pública, Brasília, DF, 6.

Santos, R. R. (2010, março). Projeto melhoria no abastecimento de combustível da frota do Estado do Ceará. Congresso CONSAD de Gestão Pública, Brasília, DF, 3.

Silva, A. M. (2016, julho). Compras Públicas e Administração Tributária: Uso da base de dados da Nota Fiscal Eletrônica na Bolsa de Compras do Amazonas. Congresso CONSAD de Gestão Pública, Brasília, DF, 9.

Silva, J. A., Rodrigues, G. L. \& Anjos, A. L. M. (2009, maio). Planejamento do consumo como instrumento de alcance dos objetivos estratégicos da Secretaria de Estado de Saúde. Congresso CONSAD de Gestão Pública, Brasília, DF, 2.

Silveira, H. F. R. Teoria e prática sobre ABC no setor público (2010, março). Congresso CONSAD de Gestão Pública, Brasília, DF, 3.

Soares, M. E. \& Moura, L. S. (2014, março). Quarteirização da manutenção da frota de veículos oficiais: o desenho do modelo de gerenciamento adotado em Minas Gerais a fim de se garantir a economicidade da contratação. Congresso CONSAD de Gestão Pública, Brasília, DF, 7.

Soares, M. E. S. \& Monteiro, V. P. (2014, março). Implementação do novo modelo de abastecimento da frota de veículos do estado de Minas Gerais. Congresso CONSAD de Gestão Pública, Brasília, DF, 7.

Souza, H. J. J. \& Freitas, D. M. M. (2016, julho). Desafios para a gestão da cadeia de suprimentos na rede de saúde do Ceará. Congresso CONSAD de Gestão Pública, Brasília, DF, 9.

Souza, W.K.A., Scalioni, L. G., Drumond, L. V. S. \& Hirle, A. L. C. (2013, abril). Gestão de fornecedores no portal de compras MG: A integração entre sistemas como forma de facilitar e ampliar o acesso das empresas ao mercado de compras governamentais. Congresso CONSAD de Gestão Pública, Brasília, DF, 9.

Teixeira, H. J., Martelanc, R. \& Prado Filho, L. P. C. (2009, maio). Dilemas e perspectivas da terceirização no setor público. Congresso CONSAD de Gestão Pública, Brasília, DF, 2.

Teixeira, H.J., Martelanc, R., Prado Filho, L. P. C. \& Salomão, S. M. (2011, maio). Logística de suprimentos e gestão de compras e contratações na Administração Pública: como fortalecer uma visão integrada. Congresso CONSAD de Gestão Pública, Brasília, DF, 4.

Teixeira, H. J., Prado Filho, L. P. C., Martelanc, R. \& Ribeiro, E. M. D (2010, março). Williamson: Prêmio Nobel de Economia 2009 e a gestão contratual na administração pública. Congresso CONSAD de Gestão Pública, Brasília, DF, 3. 
Teixeira, H. J., Silva, F. N. \& Salomão, S. M. (2014, março). A prática das compras públicas nos estados brasileiros: a inovação possível. Congresso CONSAD de Gestão Pública, Brasília, DF, 7.

Teixeira, H.J., Prado Filho, L. P. C. \& Salomão, S. M. (2016, julho). A experiência dos estados brasileiros com o pregão: avanços, limitações e perspectivas. Congresso CONSAD de Gestão Pública, Brasília, DF, 9.

Teles, J. A., Prado Filho, J. L., Zylberman, M. \& Andrade, G. (2009, maio). Resultados do uso do poder de compra do governo de Sergipe a favor das micro e pequenas empresas: Lei de Licitaçôes 6.206/07. Congresso CONSAD de Gestão Pública, Brasília, DF, 2.

Thai, K. V. (2001). Public Procurement Re-Examined. Journal of Public Procurement, 1(1), 9-50.

Thai, K. V. (2009). International public procurement: concepts and practices. In Thai, K. V. (ed.) International Handbook of Public Procurement. Boca Raton, London, N. York: CRC Press.

Thai, K. V. \& Grimm, R. (2000). Government procurement: Past and current developments, Journal of Public Budgeting, Accounting \& Financial Management, 12 (2), 231-247.

Torres, N. B., Mayer, L. \& Lunardi, P. R. S. (2013, abril). Programa Fornecer - Compras públicas para micro e pequenas empresas: licitações como política pública. Congresso CONSAD de Gestão Pública, Brasília, DF, 6.

Tridapalli, J. P., Fernandes, E. \& Machado, W.V. (2011). Gestão da cadeia de suprimento do setor público: uma alternativa para controle de gastos correntes no Brasil. Revista de Administração Pública, 45 (2), 401-33.

Vasconcelos, C. D. G. (2013, abril). Processo eletrônico de compras: celeridade e transparência. Congresso CONSAD de Gestão Pública, Brasília, DF, 6.

Vilhena, R. M. P. \& Hirle, A. L. C. (2013, abril). Gestão de compras e qualidade do gasto público: A experiência de Minas Gerais com o planejamento de compras e a integração da gestão de compras à gestão orçamentária. Congresso CONSAD de Gestão Pública, Brasília, DF, 6.

Walker, H., Essig, M., Schotanus, F., Kivistö, T. (2007). Co-operative purchasing in the public sector. In L. Knight, C. Harland, J. Telgen, K.V. Thai, G. Callender \& K. Mcken, K. (eds). Public Procurement: International cases and commentary, p. 325-342. London/N. York: Routledge.

CC BY-NC-ND 